\title{
Detecting and Correcting Speech Repairs
}

\author{
Peter Heeman and James Allen \\ Department of Computer Science \\ University of Rochester \\ Rochester, New York, 14627 \\ $\left\{\right.$ heeman, james\} $e_{c s}$. rochester.edu
}

\begin{abstract}
Interactive spoken dialog provides many new challenges for spoken language systems. One of the most critical is the prevalence of speech repairs. This paper presents an algorithm that detects and corrects speech repairs based on finding the repair pattern. The repair pattern is built by finding word matches and word replacements, and identifying fragments and editing terms. Rather than using a set of prebuilt templates, we build the pattern on the fly. In a fair test, our method, when combined with a statistical model to filter possible repairs, was successful at detecting and correcting $80 \%$ of the repairs, without using prosodic information or a parser.
\end{abstract}

\section{Introduction}

Interactive spoken dialog provides many new challenges for spoken language systems. One of the most critical is the prevalence of speech repairs. Speech repairs are dysfluencies where some of the words that the speaker utters need to be removed in order to correctly understand the speaker's meaning. These repairs can be divided into three types: fresh starts, modifications, and abridged. A fresh start is where the speaker abandons what she was saying and starts again.

the current plan is we take - okay let's say we start with the bananas (d91-2.2 utt 105)

A modification repair is where the speech-repair modifies what was said before.

after the orange juice is at - the oranges are at the OJ factory (d93-19.3 utt59)

An abridged repair is where the repair consists solely of a fragment and/or editing terms.

we need to - um manage to get the bananas to Dansville more quickly (d93-14.3 utt50)

These examples also illustrate how speech repairs can be divided into three intervals: the removed text, the editing terms, and the resumed text (cf. Levelt, (1983), Nakatani and Hirschberg, (1993)). The removed text, which might end in a word fragment, is the text that the speaker intends to replace. The end of the removed text is called the interruption point, which is marked in the above examples as "-". This is then followed by editing terms, which can either be filled pauses, such as "um", "uh", and "er", or cue phrases, such as "I mean", "I guess", and "well". The last interval is the resumed text, the text that is intended to replace the removed text. (All three intervals need not be present in a given speech repair.) In order to correct a speech repair, the removed text and the editing terms need to be deleted in order to determine what the speaker intends to say. ${ }^{1}$

In our corpus of problem solving dialogs, $25 \%$ of turns contain at least one repair, $67 \%$ of repairs occur with at least one other repair in the turn, and repairs in the same turn occur on average within 6 words of each other. As a result, no spoken language system will perform well without an effective way to detect and correct speech repairs.

We propose that most speech repairs can be detected and corrected using only local clues-it should not be necessary to test the syntactic or semantic well-formedness of the entire utterance. People do not seem to have problems comprehending speech repairs as they occur, and seem to have no problem even when multiple repairs occur in the same utterance. So, it should be possible to construct an algorithm that runs on-line, processing the input a word at a time, and committing to whether a string of words is a repair by the end of the string. Such an algorithm could precede a parser, or even operate in lockstep with it.

An ulterior motive for not using higher level syntactic or semantic knowledge is that the coverage of parsers and semantic interpreters is not sufficient for unrestricted dialogs. Recently, Dowding et al. (1993) reported syntactic and semantic coverage of $86 \%$ for the DARPA Airline reservation corpus (Dowding et al., 1993). Unrestricted dialogs will present even more difficulties; not only will the speech be less grammatical, but there is also the problem of segmenting the dialog into utterance units (cf. Wang and Hirschberg, 1992). If speech repairs can be detected and corrected before parsing and semantic interpretation, this should simplify those modules as well as make them more robust.

In this paper, we present an algorithm that detects and corrects modification and abridged speech repairs without doing syntactic and semantic processing. The algorithm determines the text that needs to be removed by building a repair pattern, based on identification of word fragments, editing

\footnotetext{
${ }^{1}$ The removed text and editing terms might still contain pragmatic information, as the following example displays, "Peter was ... well ... he was fired.
} 
terms, and word correspondences between the removed and the resumed text (cf. Bear, Dowding and Shriberg, 1992). The resulting potential repairs are then passed to a statistical model that judges the proposal as either fluent speech or an actual repair.

\section{Previous Work}

Several different strategies have been discussed in the literature for detecting and correcting speech repairs. A way to compare the effectiveness of these approaches is to look at their recall and precision rates. For detecting repairs, the recall rate is the number of correctly detected repairs compared to the number of repairs, and the precision rate is the number of detected repairs compared to the number of detections (including false positives). But the true measures of success are the correction rates. Correction recall is the number of repairs that were properly corrected compared to the number of repairs. Correction precision is the number of repairs that were properly corrected compared to the total number of corrections.

Levelt (1983) hypothesized that listeners can use the following rules for determining the extent of the removed text (he did not address how a repair could be detected). If the last word before the interruption is of the same category as the word before, then delete the last word before the interruption. Otherwise, find the closest word prior to the interruption that is the same as the first word after the interruption. That word is the start of the removed text. Levelt found that this strategy would work for $50 \%$ of all repairs (including fresh starts), get $2 \%$ wrong, and have no comment for the remaining $48 \% .^{2}$ In addition, Levelt showed that different editing terms make different predictions about whether a repair is a fresh start or not. For instance, "uh" strongly signals an abridged or modification repair, whereas a word like "sorry" signals a fresh start.

Hindle (1983) addressed the problem of correcting selfrepairs by adding rules to a deterministic parser that would remove the necessary text. Hindle assumed the presence of an edit signal that would mark the interruption point, and was able to achieve a recall rate of $97 \%$ in finding the correct repair. For modification repairs, Hindle used three rules for "expuncting" text. The first rule "is essentially a nonsyntactic rule" that matches repetitions (of any length); the second matches repeated constituents, both complete; and the third, matches repeated constituents, in which the first is not complete, but the second is.

However, Hindle's results are difficult to translate into actual performance. First, his parsing strategy depends upon the "successful disambiguation of the syntactic categories." Although syntactic categories can be determined quite well by their local context (as is needed by a deterministic parser), Hindle admits that "[self-repair], by its nature, disrupts the local context." Second, Hindle's algorithm depends on the presence of an edit signal; so far, however, the abrupt cut-off

\footnotetext{
${ }^{2}$ Levelt claims (pg. 92) that the hearer can apply his strategy safely for $52 \%$ of all repairs, but this figure includes the $2 \%$ that the hearer would get wrong.
}

that some have suggested signals the repair (cf. Labov, 1966) has been difficult to find, and it is unlikely to be represented as a binary feature (cf. Nakatani and Hirschberg, 1993).

The SRI group (Bear et al., 1992) employed simple pattern matching techniques for detecting and correcting modification repairs. ${ }^{3}$ For detection, they were able to achieve a recall rate of $76 \%$, and a precision of $62 \%$, and they were able to find the correct repair $57 \%$ of the time, leading to an overall correction recall of $43 \%$ and correction precision of $50 \%$. They also tried combining syntactic and semantic knowledge in a "parser-first" approach-first try to parse the input and if that fails, invoke repair strategies based on word patterns in the input. In a test set containing 26 repairs (Dowding et al., 1993), they obtained a detection recall rate of $42 \%$ and a precision of $84.6 \%$; for correction, they obtained a recall rate of $30 \%$ and a recall rate of $62 \%$.

Nakatani and Hirschberg (1993) investigated using acoustic information to detect the interruption point of speech repairs. In their corpus, $74 \%$ of all repairs are marked by a word fragment. Using hand-transcribed prosodic annotations, they trained a classifier on a 172 utterance training set to identify the interruption point (each utterance contained at least one repair). On a test set of 186 utterances each containing at least one repair, they obtained a recall rate of $83.4 \%$ and a precision of $93.9 \%$ in detecting speech repairs. The clues that they found relevant were duration of pause between words, presence of fragments, and lexical matching within a window of three words. However, they do not address the problem of determining the correction or distinguishing modification repairs from abridged repairs.

Young and Matessa (Young and Matessa, 1991) have also done work in this area. In their approach, speech repairs are corrected after a opportunistic case-frame parser analyzes the utterance. Their system looks for parts of the input utterance that were not used by the parser, and then uses semantic and pragmatic knowledge (of the limited domain) to correct the interpretation.

\section{The Corpus}

As part of the TRAINS project (Allen and Schubert, 1991), which is a long term research project to build a conversationally proficient planning assistant, we are collecting a corpus of problem solving dialogs. The dialogs involve two participants, one who is playing the role of a user and has a certain task to accomplish, and another, who is playing the role of the system by acting as a planning assistant. ${ }^{4}$ The entire corpus consists of 112 dialogs totaling almost eight hours in length and containing about 62,000 words, 6300 speaker turns, and 40 different speakers. These dialogs have been segmented into utterance files (cf. Heeman and Allen, 1994b); words

\footnotetext{
${ }^{3}$ They referred to modification repairs as nontrivial repairs, and to abridged repairs as trivial repairs; however, these terms are misleading. Consider the utterance "send it back to Elmira uh to make OJ". Determining that the corrected text should be "send it back to Elmira to make OJ" rather than "send it back to make OJ" is non trivial.

${ }^{4}$ Gross, Allen and Traum (1992) discuss the manner in which the first set of dialogues were collected, and provide transcriptions.
} 
have been transcribed and the speech repairs have been annotated. For a training set, we use 40 of the dialogs, consisting of 24,000 words, 725 modification and abridged repairs, and 13 speakers; and for testing, 7 of the dialogs, consisting of 5800 words, 142 modification and abridged repairs, and seven speakers, none of which were included in the training set.

The speech repairs in the dialog corpus have been handannotated. There is typically a correspondence between the removed text and the resumed text, and following Bear, Dowding and Shriberg (1992), we annotate this using the labels $\mathbf{m}$ for word matching and $\mathbf{r}$ for word replacements (words of the same syntactic category). Each pair is given a unique index. Other words in the removed text and resumed text are annotated with an $\mathbf{x}$. Also, editing terms (filled pauses and clue words) are labeled with et, and the moment of interruption with int, which will occur before any editing terms associated with the repair, and after the fragment, if present. (Further details of this scheme can be found in (Heeman and Allen, 1994a).) Below is a sample annotation, with removed text "go to oran-", editing term "um", and resumed text "go to" (d93-14.2 utt60).

gol tol oran-| um| go| tol Corning $\mathrm{m} 1|\mathrm{~m} 2| \mathrm{x} \mid$ int $\mid$ et $|\mathrm{m} 1| \mathrm{m} 2 \mid$

A speech repair can also be characterized by its repair pattern, which is a string that consists of the repair labels (word fragments are labeled as -, the interruption point by a period, and editing terms by e). The repair pattern for the example is $\mathbf{~ m m - . e m m}$.

\section{Repair Indicators}

In order to correct speech repairs, we first need to detect them. If we were using prosodic information, we could focus on the actual interruption point (cf. Nakatani and Hirschberg, 1993); however, we are restricting ourselves to lexical clues, and so need to be more lenient.

Table 1 gives a breakdown of the modification speech repairs and the abridged repairs, based on the handannotations. ${ }^{5}$ Modification repairs are broken down into four groups, single word repetitions, multiple word repetitions, one word replacing another, and others. Also, the percentage of each type of repair that include fragments and editing terms is given.

This table shows that strictly looking for the presence of fragments and editing terms will miss at least $41 \%$ of speech repairs. So, we need to look at word correspondences in order to get better coverage of our repairs. In order to keep the false positive rate down, we restrict ourselves to the following types of word correspondences: (1) word matching with at most three intervening words, denoted by $\mathbf{m}-\mathbf{m}$; (2) two adjacent words matching two others with at most 6 words

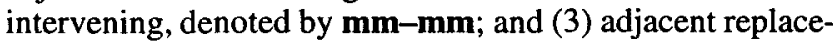
ment, denoted by rr. Table 2 the number of repairs in the

\footnotetext{
${ }^{5}$ Eight repairs were excluded from this analysis. These repairs could not be automatically separated from other repairs that overlapped with them.
}

\begin{tabular}{|l|c|c|c|}
\hline & Total & with & with Edit \\
& Frag. & Term \\
\hline Modification Repair & 450 & $14.7 \%$ & $19.3 \%$ \\
Word Repetition & 179 & $16.2 \%$ & $16.2 \%$ \\
Larger Repetition & 58 & $17.2 \%$ & $19.0 \%$ \\
Word Replacement & 72 & $4.2 \%$ & $13.9 \%$ \\
Other & 141 & $17.0 \%$ & $26.2 \%$ \\
Abridged Repair & 267 & $46.4 \%$ & $54.3 \%$ \\
\hline Total & 717 & $26.5 \%$ & $32.4 \%$ \\
\hline
\end{tabular}

Table 1: Occurrence of Types of Repairs

training corpus that can be deleted by each clue, based on the hand-annotations. For each clue, we give the number of repairs that it will detect in the first column. In the next three columns, we give a breakdown of these numbers in terms of how many clues apply. As the table shows, most repairs are signal by only one of the 3 clues.

\begin{tabular}{|c|c|c|c|c|c|}
\hline & \multicolumn{2}{|c|}{ Total } & 1 clue & 2 clues & 3 clues \\
\hline Fragment & \multicolumn{2}{|c|}{190} & $\overline{127}$ & $\overline{\overline{58}}$ & $\overline{\overline{5}}$ \\
\hline Editing Terms & \multicolumn{2}{|c|}{232} & 164 & $\overline{63}$ & 5 \\
\hline $\mathrm{m}-\mathrm{m}$ & 331 & \multirow{3}{*}{412} & \multirow{3}{*}{296} & \multirow{3}{*}{111} & \multirow{3}{*}{5} \\
\hline $\mathrm{mm}-\mathrm{mm}$ & 94 & & & & \\
\hline rr & 59 & & & & \\
\hline others & \multicolumn{2}{|c|}{9} & n.a. & n.a. & n.a. \\
\hline Total & \multicolumn{2}{|c|}{717} & 587 & 116 & 5 \\
\hline
\end{tabular}

Table 2: Repair Indicators

Although the $\mathbf{m}-\mathbf{m}$ clue and $\mathbf{m m}-\mathbf{m m}$ clue do not precisely locate the interruption point, we can, by using simple lexical clues, detect $97.7 \%$ (708/725) of all the repairs. But, we still will have a problem with false positives, and detecting the extent of the repair.

\section{Determining the Correction}

Based on the work done at SRI (Bear, Dowding and Shriberg, 1992), we next looked at the speech repair patterns in our annotated training corpus. If we can automatically determine the pattern, then the deletion of the removed text along with the editing terms gives the correction. Since the size of the pattern can be quite large, especially when editing terms and word fragments are added in, the number of possible templates becomes very large. In our training corpus of 450 modification repairs, we found 72 different patterns (not including variations due to editing terms and fragments). All patterns with at least 2 occurrences are listed in table 3.

\section{Adding to the Pattern}

Rather than doing template matching, we build the repair pattern on the fly. When a possible repair is detected, the detection itself puts constraints on the repair pattern. For instance, if we detect a word fragment, the location of the fragment limits the extent of the editing terms. It also limits 


\begin{tabular}{|l|r||l|l||}
\hline m.m & 179 & mmx.mm & 4 \\
r.r & 72 & mrm.mrm & 3 \\
mm.mm & 41 & mmmr.mmmr & 3 \\
mr.mr & 17 & mm.mxm & 3 \\
mx.m & 15 & r.xr & 2 \\
mmm.mmm & 14 & mxxx.m & 2 \\
rm.rm & 12 & mx.mx & 2 \\
m.xm & 6 & mmrm.mmrm & 2 \\
mmr.mmr & 5 & mmmx.mmm & 2 \\
m.xxm & 5 & mmmm.mmmm & 2 \\
x.xx & 4 & m.mx & 2 \\
x. & 4 & & \\
\hline
\end{tabular}

Table 3: Repair Patterns and Occurrences

the extent of the resumed text and removed text, and so on restricts word correspondences that can be part of the repair.

In this section, we present the rules we use for building repair patterns. These rules not only limit the search space, but more importantly, are intended to keep the number of false positives as low as possible, by capturing a notion of 'well-formness' for speech repairs.

The four rules listed below follow from the model of repairs that we presented in the introduction. They capture how a repair is made up of three intervals-the removed text, which can end in a word fragment, possible editing terms, and the resumed text-and how the interruption point is follows the removed text and precedes the editing terms.

1. Editing terms must be adjacent.

2. Editing terms must immediately follow the interruption point.

3. A fragment, if present, must immediately precede the interruption point.

4. Word correspondences must straddle the interruption point and can not be marked on a word labeled as an editing term or fragment.

The above rules alone do not restrict the possible word correspondences enough. Based on an analysis of the handcoded repairs in the training corpus, we propose the following additional rules.

Rule (5) captures the regularity that word correspondences of a modification repair are rarely, if ever, embedded in each other. Consider the following exception.

how would that - how long that would take

In this example, the word correspondence involving "that" is embedded inside of the correspondence on "would". The speaker actually made a uncorrected speech error (and so not a speech repair) in the resumed text, for he should have said "how long would that take." Without this ungrammaticality, the two correspondences would not have been embedded, and so would not be in conflict with the following rule.

5. Word correspondences must be cross-serial; a word correspondence cannot be embedded inside of another correspondence.
The next rule is used to limit the application of word correspondences when no correspondences are yet in the repair pattern. In this case, the repair would have been detected by the presence of a fragment or editing terms. This rule is intended to prevent spurious word correspondences from being added to the repair. For instance in the following example, the correspondence between the two instances of " $T$ " is spurious, since the second "I" in fact replaces "we".

I think we need to uh I need

So, when no correspondences are yet included in the repair, the number of intervening words needs to be limited. From our test corpus, we have found that 3 intervening words, excluding fragments and editing terms is sufficient.

6. If there are no other word correspondences, there can only be 3 intervening words, excluding fragments and editing terms, between the first part and the second part of the correspondence.

The next two rules restrict the distance between two word correspondences. Figure 1 shows the distance between two word correspondences, indexed by $i$ and $j$. The intervals $x$ and $y$ are sequences of the words that occur between the marked words in the removed text and in the resumed text, respectively. The word correspondences of interest are those that are adjacent, in order words, the ones that have no labeled words in the $x$ and $y$ intervals.

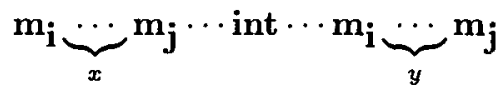

Figure 1: Distance between correspondences

For two adjacent word correspondences, Rule (7) ensures that there is at most 4 intervening words in the removed text, and Rule (8) ensures that there are at most 4 intervening words in the resumed text.

7. In the removed text, two adjacent matches can have at most 4 intervening words $(|x| \leq 4)$.

8. In the resumed text, two adjacent matches can have at most 4 intervening words $(|y| \leq 4)$.

The next rule, Rule (9), is used to capture the regularity that words are rarely dropped from the removed text, instead they tend to be replaced.

9. For two adjacent matches, the number of intervening words in the removed text can be at most one more than the number of intervening words in the resumed text $(|x| \leq|y|+1)$.

The last rule, Rule (10), is used to restrict word replacements. From an analysis of our corpus, we found that word replacement correspondences are rarely isolated from other word correspondences.

10. A word replacement (except those added by the detection clues) must either only have fragments and editing terms between the two words that it marks, or there must be a word correspondence in which there are no intervening words in either the removed text or the resumed text $(x=y=0)$. 


\section{An Example}

To illustrate the above set of well-formedness constraints on repair patterns, consider the example given above "I think we need to - uh I need." The detection clues will mark the word "uh" as being a possible editing term, giving the partial pattern given below.

I think we need to uh| I need

Now let's consider the two instances of " $I$ ". Adding this correspondence to the repair pattern will violate Rule (6), since there are four intervening words, excluding the editing terms. The correspondence between the two instances of 'need' is acceptable though, since it straddles the editing term, and there are only two intervening words between the corresponding words, excluding editing terms.

Even with the correspondence between the two instances of 'need', the matching between the 'I's still cannot be added. There are 2 intervening words between "I" and "need" in the removed text, but none in the resumed side, so this correspondence violates Rule (9). The word replacement of "we" by the second instance of "I", does not violate any of the rules, including Rule (10), so it is added, resulting in the following labeling.

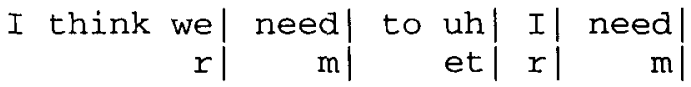

\section{Algorithm}

Our algorithm for labeling potential repair patterns encodes the assumption that speech repairs can be processed one at a time. The algorithm runs in lockstep with a part-of-speech tagger (Church, 1988), which is used for deciding possible word replacements. Words are fed in one at a time. The detection clues are checked first. If one of them succeeds, and there is not a repair being processed, then a new repair pattern is started. Otherwise, if the clue is consistent with the current repair pattern, then the pattern is updated; otherwise, the current one is sent off to be judged, and a new repair pattern is started.

When a new repair is started, a search is made to see if any of the text can contribute word correspondences to the repair. Likewise, if there is currently a repair being built, a search is made to see if there is a suitable word correspondence for the current word. Anytime a correspondence is found, a search is made for any additional correspondences that it might sanction.

Since there might be a conflict between two possible correspondences that can be added to a labeling, the one that involves the most recent pair of words is preferred. For instance, in the example above, the correspondence between the second instance of " $I$ " and "we" is prefered over the correspondence between the second instance of " $\mathrm{I}$ " and the first.

The last issue to account for is the judging of a potential repair. If the labeling consists of just cue phrases, then it is judged as not being a repair. ${ }^{6}$ Otherwise, if the point of

\footnotetext{
${ }^{6}$ This prevents phrases such as "I guess" from being marked as
}

interruption of the potential repair is uniquely determined, then it is taken as a repair. This will be the case if there is at least one editing term, a word fragment, or there are no unaccounted for words between the last removed text part of the last correspondence and the resumed text part of the first correspondence.

\section{Results of Pattern Building}

The input to the algorithm is the word transcriptions, augmented with turn-taking markers. Since we are not trying to account for fresh starts, break points are put in to denote the cancel, and its editing terms are deleted (this is done to prevent the algorithm from trying to annotate the fresh start as a repair). The speech is not marked with any intonational information, nor is any form of punctuation inserted. The results are given in Table 4.

\begin{tabular}{|l|c|c|}
\hline & $\begin{array}{c}\text { Training } \\
\text { Set }\end{array}$ & $\begin{array}{c}\text { Test } \\
\text { Set }\end{array}$ \\
\hline Detection Recall & $94.9 \%$ & $91.5 \%$ \\
Detection Precision & $55.8 \%$ & $45.3 \%$ \\
Correction Recall & $89.2 \%$ & $85.9 \%$ \\
Correction Precision & $52.4 \%$ & $42.5 \%$ \\
\hline
\end{tabular}

Table 4: Results of Pattern Matching

The pattern builder gives many false positives in detecting speech repairs due to word correspondences in fluent speech being mis-interpreted is evidence of a modification repair. Also, in correcting the repairs, word correspondences across an abridged repair cause the abridged repair to be interpreted as a modification repair, thus lowering the correction recall rate. ${ }^{7}$ For example, the following abridged repair has two spurious word correspondences, between "need to" and "manage to".

we need to - um manage to get the bananas to Dansville more quickly

This spurious word correspondence will cause the pattern builder to hypothesize that this is a modification repair, and so propose the wrong correction.

\section{Adding A Statistical Filter}

We make use of a part-of-speech tagger to not only determine part-of-speech categories (used for deciding possible word replacements), but also to judge modification repairs that are proposed by the pattern builder. For modification repairs, the category transition probabilities from the last word of the removed text to the first word of the resumed text have a different distribution than category transitions for fluent speech. So, by giving these distributions to the part-ofspeech tagger (obtained from our test corpus), the tagger can decide if a transition signals a modification repair or not.

editing terms when they have a sentential meanings, as in "I guess we should load the oranges."

${ }^{7}$ About half of the difference between the detection recall rate and the correction recall rate is due to abridged repairs being misclassified as modification repairs. 
Part-of-speech tagging is the process of assigning to a word the category that is most probable given the sentential context (Church, 1988). The sentential context is typically approximated by only a set number of previous categories, usually one or two. Good part-of-speech results can be obtained using only the preceding category (Weischedel et al., 1993), which is what we will be using. In this case, the number of states of the Markov model will be $N$, where $N$ is the number of tags. By using the Viterbi algorithm, the part-of-speech tags that lead to the maximum probability path can be found in linear time.

Figure 2 gives a simplified view of a Markov model for part-of-speech tagging, where $C_{i}$ is a possible category for the $i$ th word, $w_{i}$, and $C_{i+1}$ is a possible category for word $w_{i+1}$. The category transition probability is simply the probability of category $C_{i+1}$ following category $C_{i}$, which is written as $P\left(C_{i+1} \mid C_{i}\right)$. The probability of word $w_{i+1}$ given category $C_{i+1}$ is $P\left(w_{i+1} \mid C_{i+1}\right)$. The category assignment that maximizes the product of these probabilities is taken to be the best category assignment.

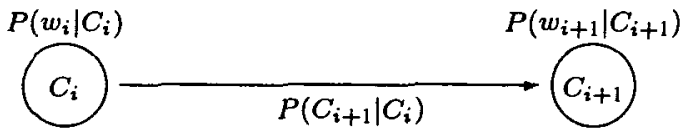

Figure 2: Markov Model of Part-of-Speech Tagging

To incorporate knowledge about modification repairs, we let $R_{i}$ be a variable that indicates whether the transition from word $w_{i}$ to $w_{i+1}$ contains the interruption point of a modification repair. Rather than tag each word, $w_{i}$, with just a category, $C_{i}$, we will tag it with $R_{i-1} C_{i}$, the category and the presence of a modification repair. So, we will need the following probabilities, $P\left(R_{i} C_{i+1} \mid R_{i-1} C_{i}\right)$ and $P\left(w_{i} \mid R_{i-1} C_{i}\right)$. To keep the model simple, and ease problems with sparse data, we make several independence assumptions. By assuming that $R_{i-1}$ and $R_{i} C_{i+1}$ are independent, given $C_{i}$, we can simplify the first probability to $P\left(R_{i} \mid C_{i}\right) * P\left(C_{i+1} \mid C_{i} R_{i}\right)$; and by assuming that $R_{i-1}$ and $w_{i}$ are independent, given $C_{i}$, we can simplify the second one to $P\left(w_{i} \mid C_{i}\right)$. The model that results from this is given in Figure 3. As can be seen, these manipulations allow us to view the problem as tagging null tokens between words as either the interruption point of a modification repair, $R_{i}=\tau_{i}$, or as fluent speech, $R_{i}=\phi_{i}$.

Modification repairs can be signaled by other indicators than just syntactic anomalies. For instance, word matches, editing terms, and word fragments also indicate their presence. This information can be added in by viewing the presence of such clues as the 'word' that is tagged by the repair indicator $R_{i}$. By assuming that these clues are independent, given the presence of a modification repair, we can simply use the product of the individual probabilities. So, the repair state would have an output probability of $P\left(F_{i} \mid R_{i}\right) * P\left(E_{i} \mid R_{i}\right) * P\left(M_{i} \mid R_{i}\right)$, where $F_{i}, E_{i}$, and $M_{i}$ are random variables ranging over fragments, editing terms, types of word matches, respectively. So for instance, the

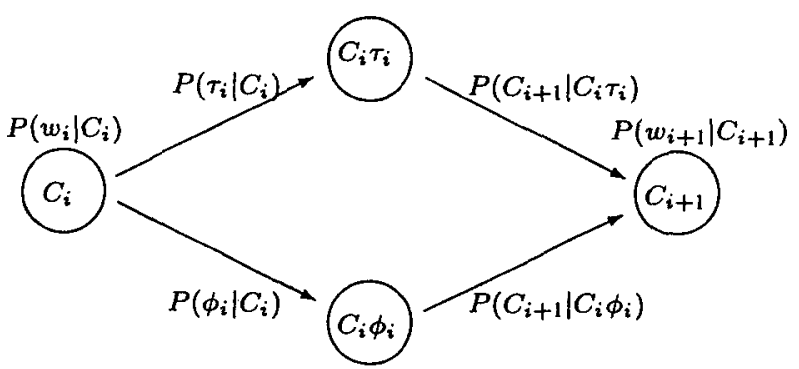

Figure 3: Statistical Model of Speech Repairs

model can account for how "uh" is more likely to signal a modification repair than "um". Further details are given in Heeman and Allen (1994c).

\section{Overall Results}

The pattern builder on its own gives many false positives due to word correspondences in fluent speech being misinterpreted evidence of a modification repair, and due to word correspondences across an abridged repair causing the abridged repair to be interpreted as a modification repair. This results in an overall correction recall rate of $86 \%$ and a precision rate of $43 \%$. However, the real result comes from coupling the pattern builder with the decision routine, which will eliminate most of the false positives.

Potential repairs are divided into two groups. The first includes abridged repairs and modification repairs involving only word repetitions. These are classified as repairs outright. The Rest of the modification repairs are judged by the statistical model. Any potential repair that it rejects, but which contains a word fragment or filled pause is accepted as an abridged repair. Table 5 gives the results of the combined approach on the training and test sets.

\begin{tabular}{|l|c|c|}
\hline & $\begin{array}{c}\text { Training } \\
\text { Corpus }\end{array}$ & $\begin{array}{c}\text { Test } \\
\text { Corpus }\end{array}$ \\
\hline Detection & & \\
Recall & $91 \%$ & $83 \%$ \\
Precision & $96 \%$ & $89 \%$ \\
\hline Correction & & \\
Recall & $88 \%$ & $80 \%$ \\
Precision & $93 \%$ & $86 \%$ \\
\hline
\end{tabular}

Table 5: Overall Results

Comparing our results to others that have been reported in the literature must be done with caution. Such a comparison is limited due to differences in both the type of repairs that are being studied and in the datasets used for drawing results. Bear, Dowding, and Shriberg (1992) use the ATIS corpus, which is a collection of queries made to an automated airline reservation system. As stated earlier, they removed all utterances that contained abridged repairs. For detection they obtained a recall rate of $76 \%$ and a precision of $62 \%$, and for correction, a recall rate of $43 \%$ and a precision of $50 \%$. It is not clear whether their results would be better or worse if 
abridged repairs were included. Dowding et al. (1993) used a similar setup for their data. As part of a complete system, they obtained a detection recall rate of $42 \%$ and a precision of $85 \%$; and for correction, a recall rate of $30 \%$ and a precision of $62 \%$. Lastly, Nakatani and Hirschberg (1993) also used the ATIS corpus, but in this case, focused only on detection, but detection of all three types of repairs. However, their test corpus consisted entirely of utterances that contained at least one repair. This makes it hard to evaluate their results, reporting a detection recall rate of $83 \%$ and precision of $94 \%$. Testing on an entire corpus would clearly decrease their precision. As for our own data, we used a corpus of natural dialogues that were segmented only by speaker turns, not by individual utterances, and we focused on modification repairs and abridged repairs, with fresh starts being marked in the input so as not to cause interference in detecting the other two types.

The performance of our algorithm for correction is significantly better than other previously reported work, with a recall rate of $80.2 \%$ and a precision rate of $86.4 \%$ on a fair test. While Nakatani and Hirschberg report comparable detection rates, and Hindle reports better correction rates, neither of these researchers attack the complete problem of both detection and correction. Both of them also depend on externally supplied annotations not automatically derived from the input. As for the SRI work, their parser-first strategy and simple repair patterns cause their rates to be much lower than ours. A lot of speech repairs do not look ill-formed, such as "and a boxcar of - and a tanker of OJ", and "and bring - and then bring that orange juice," and are mainly signaled by either lexical or acoustic clues.

\section{Overlapping Repairs}

Our algorithm is also novel in that it handles overlapping repairs. Two repairs overlap if part of the text is used in both repairs. Such repairs occur fairly frequently in our corpus, and for the most part, our method of processing repairs, even overlapping ones, in a sequential fashion appears successful. Out of the 725 modification and abridged repairs in the training corpus, 164 of them are overlapping repairs, and our algorithm is able to detect and correct $86.6 \%$ of them, which is just slightly less than the correction recall rate for all modification and abridged repairs in the entire training corpus.

Consider the following example (d93-14.2 utt26), which contains four speech repairs, with the last one overlapping the first three.

and pick up um the en- I guess the entire um p- pick up the load of oranges at Corning

The algorithm is fed one word at a time. When it encounters the first "um", the detection rule for editing terms gets activated, and so a repair pattern is started, with "um" being labeled as an editing term. The algorithm then processes the word "the", for which it can find no suitable correspondences. Next is the fragment "en-". This causes the detection rule for fragments to fire. Since this fragment comes after the editing term in the repair being built, adding it to the repair would violate Rule (2) and Rule (3). So, the algorithm must finish with the current repair, the one involving "um". Since this consists of just a filled pause, it is judged as being an actual repair.

Now that the alogrithm is finished with the repair involving "um", it can move on to the next one, the one signaled by the fragment "en-". The next words that are encountered are "I guess", which get labeled as an editing phrase. The next token is the word "the", for which the algorithm finds a word correspondence with the previous instance of "the". At this point, it realizes that the repair is complete (since there is a word correspondence and all words between the first marked word and the last are accounted for) and so sends it off to be judged by the statistical model. The model tags it as a repair. Deleting the removed text and the editing terms indicated by the labeling results in the following, with the algorithm currently processing "the".

and pick up the entire um p- pick up the load of oranges at Corning

Continuing on, the next potential repair is triggered by the presence of "um", which is labeled as an editing term. The next token encountered, a fragment, also indicates a potential repair, but adding it to the labeling will violate Rule (2) and Rule (3). So, the pattern builder is forced to finish up with the potential repair involving "um". Since this consists of just a filled pause, it is accepted. This leaves us with the following text, with the algorithm currently processing " $p$-", which it has marked as a fragment.

and pick up the entire p- pick up the load of oranges at Corning

The next word it encounters is "pick". This word is too far from the preceding "pick" to allow this correspondence to be added. However, the detection clue $\mathbf{m m - m m}$ does fire, due to the matching of the pair of adjacent words "pick up". This clue is consistent with "p-" being marked as the word fragment of the repair, and so these correspondences are added. The next token encountered is "the", and the correspondence for it is found. Then "load" is processed, but no correspondence is found for it, nor for the remaining words. So, the repair pattern that is built contains an unlabeled token, namely "entire". But due to the presence of the word fragment, the interruption point can be determined. The repair pattern is set off to be judged, which tags it as a repair. This leaves the following text not labeled as the removed text nor as the editing terms of a repair.

and pick up the load of oranges at Corning

Due to the sequential processing of the algorithm and its ability to commit to a repair without seeing the entire utterance, overlapping repairs do not pose a major problem.

Some overlapping repairs can cause problems however. Problems can occur when word correspondences are attributed to the wrong repair. Consider the following example (d93-15.2 utt46).

you have w- one you have two boxcar

This utterance contains two speech repairs, the first is the replacement of "w-" by "one", and the second the replacement of "you have one" by "you have two". Since no analysis of fragments is done, the correspondence between "w-" and 
"one" is not detected. So, our greedy algorithm decides that the repair after " $w$-" also contains the word matches for "you" and "have", and that the occurrence of "one" after the "w-" is an inserted word. Due to the presence of the partial and the word matching, the statistical model accepts this proposal, which leads to the erroneous correction of "one you have two boxcars," which blocks the subsequent repair from being found.

\section{Conclusion}

This paper described a method of locally detecting and correction modification and abridged speech repairs. Our work shows that a large percentage of speech repairs can be resolved prior to parsing. Our algorithm assumes that the speech recognizer produces a sequence of words and identifies the presence of word fragments. With the exception of identifying fresh starts, all other processing is automatic and does not require additional hand-tailored transcription. We will be incorporating this method of detecting and correcting speech repairs into the next version of the TRAINS system, which will use spoken input.

There is an interesting question as to how good the performance can get before a parser is required in the process. Clearly, some examples require a parser. For instance, we can not account for the replacement of a noun phrase with a pronoun, as in "the engine can take as many um - it can take up to three loaded boxcars" without using syntactic knowledge. On the other hand, we can expect to improve on our performance significantly before requiring a parser. The scores on the training set, as indicated in table 5 , suggest that we do not have enough training data yet. In addition, we do not yet use any prosodic cues. We are currently investigating methods of automatically extracting simple prosodic measures that can be incorporated into the algorithm. Given Nakatani and Hirschberg's results, there is reason to believe that this would significantly improve our performance.

Although we did not address fresh starts, we feel that our approach of combining local information from editing terms, word fragments, and syntactic anomalies will be successful in detecting them. However, the problem lies in determining the extent of the removed text. In our corpus of spoken dialogues, the speaker might make several contributions in a turn, and without incorporating other knowledge, it is difficult to determine the extent of the text that needs to be removed. We are currently investigating approaches to automatically segment a turn into separate utterance units by using prosodic information.

\section{Acknowledgments}

We wish to thank Bin Li, Greg Mitchell, and Mia Stern for their help in both transcribing and giving us useful comments on the annotation scheme. We also wish to thank Hannah Blau, John Dowding, Elizabeth Shriberg, and David Traum for helpful comments. Funding gratefully received from the Natural Sciences and Engineering Research Council of Canada, from NSF under Grant IRI-90-13160, and from ONR/DARPA under Grant N00014-92-J-1512.

\section{References}

Allen, J. F. and Schubert, L. K. (1991). The TraINS project. Technical Report 382, Department of Computer Science, University of Rochester.

Bear, J., Dowding, J., and Shriberg, E. (1992). Integrating multiple knowledge sources for detection and correction of repairs in human-computer dialog. In Proceedings of the $30^{\text {th }}$ Annual Meeting of the Association for Computational Linguistics, pages 56-63.

Church, K. (1988). A stochastic parts program and noun phrase parser for unrestricted text. In Preceedings of the 2nd Conference on Applied Natural Language Processing, pages 136-143.

Dowding, J., Gawron, J. M., Appelt, D., Bear, J., Cherny, L., Moore, R., and Moran, D. (1993). Gemini: A natural language system for spoken-language understanding. In Proceedings of the $3 I^{\text {th }}$ Annual Meeting of the Association for Computational Linguistics, pages 54-61.

Gross, D., Allen, J., and Traum, D. (1992). The Trains 91 dialogues. Trains Technical Note 92-1, Department of Computer Science, University of Rochester.

Heeman, P. A. and Allen, J. (1994a). Annotating speech repairs. unpublished manuscript.

Heeman, P. A. and Allen, J. (1994b). Dialogue transcription tools. unpublished manuscript.

Heeman, P. A. and Allen, J. (1994c). Tagging speech repairs. In ARPA Workshop on Human Language Technology, Princeton.

Hindle, D. (1983). Deterministic parsing of syntactic nonfluencies. In Proceedings of the $21^{\text {st }}$ Annual Meeting of the Association for Computational Linguistics, pages 123-128.

Labov, W. (1966). On the grammaticality of everyday speech. Paper presented at the Linguistic Society of America Annual Meeting.

Levelt, W. J. M. (1983). Monitoring and self-repair in speech. Cognition, 14:41-104.

Nakatani, C. and Hirschberg, J. (1993). A speech-first model for repair detection and correction. In Proceedings of the $3 I^{\text {th }}$ Annual Meeting of the Association for Computational Linguistics, pages 46-53.

Wang, M. Q. and Hirschberg, J. (1992). Automatic classification of intonational phrase boundaries. Computer Speech and Language, 6:175-196.

Weischedel, R., Meteer, M., Schwartz, R., Ramshaw, L., and Palmucci, J. (1993). Coping with ambiguity and unknown words through probabilistic models. Computational Linguistics, 19(2):359-382.

Young, S. R. and Matessa, M. (1991). Using pragmatic and semantic knowledge to correct parsing of spoken language utterances. In Proceedings of the 2nd European Conference on Speech Communication and Technology (Eurospeech 9l), Genova, Italy. 